\title{
Production d'acide lactique à partir de perméat de lactosérum par fermentation continue en réacteur à membrane
}

\author{
P. BOYAVAL, Sylvie TERRE et C. CORRE \\ I.N.R.A., Laboratoire de Recherches de Technologie laitière \\ 65, rue de Saint-Brieuc, 35042 Rennes Cedex, France
}

\begin{abstract}
Résumé
La production d'acide lactique en continu à partir de perméat de lactosérum est mise en œuvre, dans un procédé continu comprenant trois opérations unitaires respectivement dans un bioréacteur, un module d'ultrafiltration et un électrodialyseur. L'ultrafiltration permet le recyclage de tout ou partie de la biomasse et la séparation des métabolites de faibles poids moléculaires dont principalement le lactate de sodium issu de la fermentation du lactose, sur laquelle il exerce une action inhibitrice. Ce produit est ensuite extrait et concentré en continu par électrodialyse. Le dispositif est testé avec une culture mixte de Lactobacillus helveticus et Streptococcus thermophilus. A $45^{\circ} \mathrm{C}$ et pH 5,5 et pour un taux de dilution de $0,77 \mathrm{~h}^{-1}$, nous obtenons une productivité de $17,3 \mathrm{~g} \cdot \mathrm{l}^{-1} \cdot \mathrm{h}^{-1}$ de lactate avec une concentration cellulaire moyenne de $40 \mathrm{~g} \cdot \mathrm{l}^{-1}$. Le couplage de l'électrodialyseur permet l'obtention d'une solution finale de $130 \mathrm{~g} \cdot \mathrm{l}^{-1} \mathrm{de}$ lactate. La stabilisation du niveau de biomasse n'est obtenue que lors de fermentations au cours desquelles une purge cellulaire permet le retrait continu de cellules. Cette opération offre en outre l'avantage de produire des ferments concentrés. La mise en place d'une boucle de recirculation au niveau de l'électrodialyseur permet de recycler tout ou partie du lactose résiduel lorsque des taux de dilution élevés sont mis en œuvre. De tels taux ne permettent pas, en effet, la bioconversion totale du lactose par les bactéries utilisées.
\end{abstract}

Mots clés : Perméat de lactosérum - Ultrafiltration - Electrodialyse - Lactose - Lactate Lactobacillus helveticus - Streptococcus thermophilus.

\section{Summary}

Continuous fermentation of sweet whey permeate for lactic acid production in a membrane bioreactor

Continuous production of lactic acid from whey permeate was studied. The used equipment consisted of a fermentation unit, an ultrafiltration module and an electrodialysis unit. Ultrafiltration allowed total or partial biomass recycling and low molecular weight compounds separation (sodium lactate or residual lactose). Sodium lactate extraction is particularly interesting because of its inhibitory effect on fermentation, it was then continuously extracted and concentrated in the electrodialysis apparatus. The equipement was tested with a mixed culture of Lactobacillus helveticus and Streptococcus thermophilus. 
At $45^{\circ} \mathrm{C}$ and $\mathrm{pH} 5.5$ and with a dilution rate of $0.77 \mathrm{~h}^{-1}$, a lactate productivity of $17.3 \mathrm{~g} \cdot \mathrm{l}^{-1} \cdot \mathrm{h}^{-1}$ was obtained with a cell density of $40 \mathrm{~g} \cdot \mathrm{l}^{-1}$. Through the coupling of the electrodialyzer, we obtained an end product containing $130 \mathrm{~g}$ of lactate per liter. We also demonstrated that it is possible to stabilize biomass by means of a continuous cellular bleeding. This operation also offers possibility for production of concentrated starters. Concerning electrodialysis, we have set up a recycling loop for the residual lactose. This system was particularly efficient, when having high dilution rates and low lactose conversion rates.

The double interest of working with medium cellular density (about $20 \mathrm{~g} \cdot \mathrm{1}^{-1}$ ) to obtain a high specific productivity (which decreases very quickly when the biomass increases) and to avoid ultrafiltration membrane fouling was described. In a technical point of view, some modifications are necessary to improve the process and in particular we actually increase ultrafiltration membrane area to obtain higher dilution rates. From an economical point of view we have outlined the importance of product quality (purity, colour, mineral content...) to define the outlets of the process.

Key words: Whey permeate - Ultrafiltration - Electrodialysis - Lactose - Lactate Lactobacillus helveticus - Streptococcus thermophilus.

\section{Introduction}

L'évolution des industries fromagères conduit à une production de lactosérum de plus en plus importante $\left(9.10^{7} \mathrm{t}\right.$ en 1985 dans le monde, CNIEL, 1986).

Le traitement par ultrafiltration sur membrane conduit à l'obtention de deux produits : un concentré de protéines qui de par ses qualités nutritionnelles et fonctionnelles trouve de nombreux et larges débouchés dans les secteurs de la seconde transformation agro-alimentaire (Bools, 1985) et de la diététique classique ou spécialisée (MAUBoIS, 1982) et un perméat caractérisé par une forte teneur en lactose ( 87 p. 100 de la substance sèche) et en sels minéraux ( 9 p. 100 de la substance sèche). Ces teneurs limitent ses débouchés soit à l'alimentation animale (celle du ruminant ou celle du porc à l'engrais) soit à l'utilisation comme substrat de fermentation.

La bioconversion du lactose contenu dans le perméat d'ultrafiltration par les levures est une réalité industrielle, tant pour la production de biomasse (Moulin et Galzy, 1976; Moulin et al., 1983) que pour la production d'alcool de bouche (Burgess et Kelly, 1979 ; Hansen, 1980).

D'autres bioconversions sont envisageables car nombre de microorganismes ont la capacité de dégrader le lactose et de le convertir en différents métabolites : méthane (COTON, 1980 ; Morris, 1982), solvants (LEE et MADDox, 1986), acides aminés (Ko et CHIPLEY, 1983), polysaccharide, xanthane (Schwartz, 1986) ou acides organiques. Parmi ces derniers, la transformation en acide lactique apparaît comme la plus prometteuse (COTON, 1980) en raison de la relative ampleur du marché mondial de cet acide (35000 tonnes) (Michelet et al., 1982) et de l'adéquation des microorganismes utilisés quotidiennement par l'industrie laitière à cette bioconversion. Les débouchés pour l'acide lactique sont en effet importants et variés. En agro-alimentaire, il est utilisé comme acidulant (boissons gazeuses, bière, cidre), conservateur (choucroute, cornichons, pain), anticristallisant (confiserie) ou acidifiant (beurre). 
En pharmacie, les lactates de calcium et de fer sont employés pour lutter contre les carences en ces deux oligo-éléments et les polymères d'acide lactique comme supports biodégradables de principes actifs sous forme d'implants. Enfin, en industrie chimique, il trouve de nombreuses applications (peintures, tannerie, mordants pour teinture, préparation de résines, etc.) (Michelet et al., 1982). Les principaux obstacles au développement de procédés biologiques dans cette voie étaient jusqu'à maintenant de deux ordres: de mauvaises productivités liées à l'inhibition de la fermentation par le lactate produit (Luedeking et Piret, 1959) et un coût d'extraction et de purification trop élevé (SMITH et al, , 1977). Un procédé performant devra donc assurer une forte productivité globale et diminuer les étapes de purification à mettre en œuvre en aval. De hautes productivités peuvent être atteintes en travaillant à haute densité cellulaire.

Deux possibilités s'offrent pour atteindre ce but : le confinement ou la recirculation de la biomasse pour lesquelles différentes techniques ont été proposées.

Il peut s'agir d'immobilisation (adsorption, inclusion ou liaison covalente) (ChAmpagne et Boyaval, 1986) ou de recirculation après floculation, centrifugation ou séparation à l'aide de membranes (Goma et Durand, 1986). La centrifugation avec recirculation du filtrat a été étudiée par RAMANATHAN et Gaudy en 1971 et Topiwala et KHOSRovi en 1978 ; elle ne fait cependant pas à l'heure actuelle l'objet d'applications industrielles. L'immobilisation a été proposée dans de nombreux travaux et a été plus particulièrement utilisée pour la production d'acide lactique. Lactobacillus delbrueckii a été immobilisé dans les billes d'alginate de calcium par STENRoos et al. (1982) et Lactobacillus casei a été inclus dans des cubes de gel (polyacrylamide ou agarose) par Tuli et al. en 1985. Cependant, les méthodes employées actuellement qui font intervenir la filtration sur membrane avec recirculation du rétentat semblent beaucoup plus prometteuses. Elles permettent en effet d'éliminer le milieu contenant le métabolite inhibiteur tout en conservant la biomasse active.

Dès les années 70, l'ultrafiltration est développée aux Etats-Unis pour le confinement des biocatalyseurs (cellules, enzymes) (Porter et Michaels, 1970 ; SonOYAma, 1968 ; Wang et al., 1970). Elle a connu ensuite de nombreuses applications avec les modules type "fibre creuse». Ceux-ci peuvent être utilisés pour confectionner des réacteurs "piston", les cellules étant alors localisées dans le support macroporeux des membranes ou dans le compartiment perméat, Mehaia et Cheryan ont réalisé ce type de réacteur pour la conversion du lactose par Kluyveromyces fragilis (1984 b) et du saccharose par Saccharomyces cerevisiae (1984 a), de même que InLOES et al. en 1983. On peut aussi utiliser l'ultrafiltre pour confiner le biocatalyseur dans le circuit rétentat. On obtient alors un réacteur parfaitement mélangé. MenaiA et Cheryan en 1986 ont exploité ce système pour la production d'acide lactique.

Deux possibilités s'offraient a priori pour l'extraction du lactate produit : l'échange d'ions ou l'électrodialyse. De nombreux auteurs s'accordent à dire que pour traiter de grandes quantités et pour une déminéralisation partielle le deuxième procédé est beaucoup mieux adapté (DE BOer et RoBBERTSEN, 1983). Cette étape d'extraction en continu peut être envisagée juste en aval de la fermentation. Cependant les résultats obtenus par HoNGo et al. (1986) ont 
montré que l'électrodialyse directe du milieu de culture s'accompagnait d'un colmatage très rapide des membranes. C'est pourquoi, nous avons finalement retenu pour notre étude un procédé couplant ultrafiltration et électrodialyse, tel que décrit par Prigent et Franco en 1984. Le bioréacteur est couplé à un module d'ultrafiltration. On obtient alors d'une part un rétentat pouvant renfermer de hautes densités cellulaires et d'autre part, un produit déjà purifié contenant acide lactique, lactose résiduel et sels minéraux. Ce produit de faible viscosité est ensuite envoyé dans un électrodialyseur où est extrait le lactate alors que le lactose non transformé peut être recyclé dans le fermenteur.

Cette technologie d'association de procédés à membranes n'a jamais été utilisée en fermentation continue. Elle offre l'avantage, notamment vis-à-vis du procédé décrit par Mehaia et CherYan (1986), de produire peu d'effluents mais très concentrés en lactate et appauvris en éléments nutritifs résiduels et en certains minéraux.

Notre objectif était la mise en cuvre, l'optimisation et, [collaboration avec l'Ecole Supérieure d'Electricité (E.S.E.) et l'Ecole Nationale Supérieure de Chimie de Rennes (E.N.S.C.R.)] la modélisation du procédé en vue de son automatisation. Nous présentons ici les principaux résultats issus d'essais préliminaires ainsi que les objectifs fixés pour la suite de notre étude.

\section{Matériels et méthodes}

\section{A. Matériel}

\section{Dispositif expérimental}

La figure 1 présente les trois principaux appareils utilisés et leur raccordement hydraulique ainsi que l'emplacement des différents capteurs employés (liaison par des raccords souples stérilisables).

a) Fermenteur. Nous utilisons un réacteur possédant un volume utile de 1,5 litre (SETRIC, type $2 \mathrm{M}$ ) muni d'un dispositif de régulation de $\mathrm{pH}$ et de température. Nous travaillons à poids de milieu constant, le fermenteur étant placé sur une balance reliée à une régulation commandant son alimentation. La vitesse d'agitation est réglée à 200 ou 300 rotations par minute selon la viscosité du milieu.

N.B. - Le couplage du fermenteur avec le circuit d'ultrafiltration nous permet de travailler avec un volume utile de fermentation de 31 .

b) Module d'ultrafiltration. Il s'agit du module de laboratoire P SV3R de la S.F.E.C. (Société de Fabrication d'Eléments Catalytiques, Bollène, France). Il est équipé de membranes minérales en oxyde de zirconium (Carbosep) de type $\mathrm{M}_{4}$ (seuil de coupure à un poids moléculaire de 20000 daltons). La surface totale de filtration est de $0,10 \mathrm{~m}^{2}$. La circulation des fluides est assurée par une pompe positive à vitesse variable (servomoteur). Le système est pourvu d'une vanne de contre-pression, ce qui permet de faire varier la 


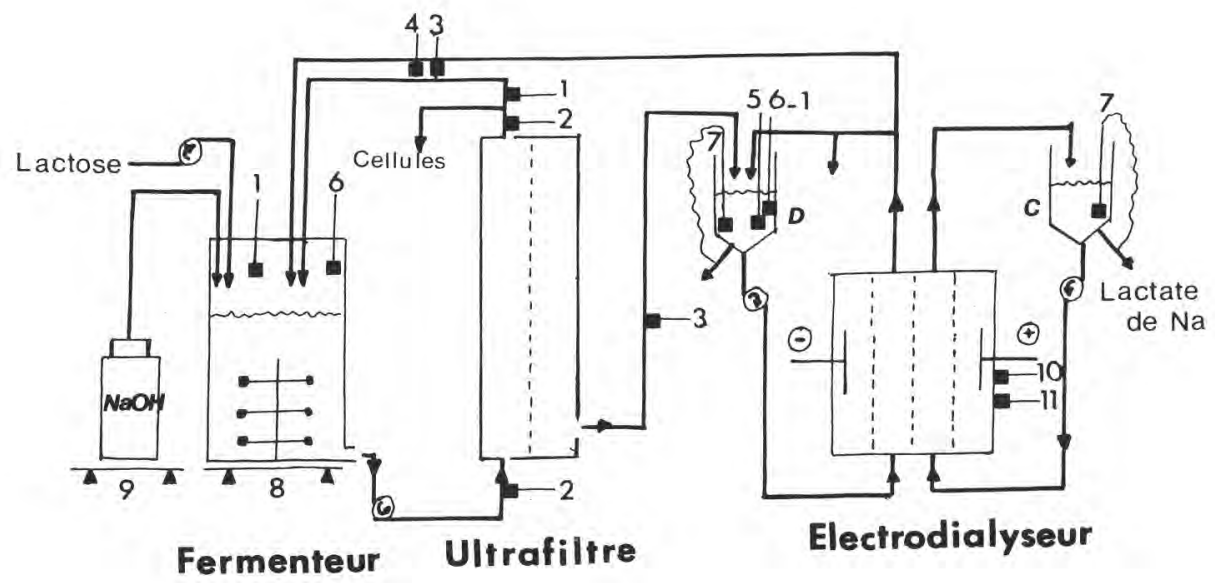

Fig. 1

Schéma hydraulique du dispositif de production de lactate et lieux d'insertion des différents capteurs.

Set-up hydraulic flow-sheet for lactate production. Implantation of the different sensors.

1 Température - Temperature.

2 Pression - Pressure.

3 Débit - Flow-rate.

4 Densité optique - Optical density.

5 Conductivité - Conductivity.

$6 \mathrm{pH}-\mathrm{pH}$.

7 Niveau - Level.

8-9 Poids - Weight.

10 Intensité - Intensity.

11 d.d.p. - Voltage.

pression indépendamment de la vitesse de balayage des fluides sur la membrane. Un échangeur de chaleur, couplé au module d'UF, assure la régulation de température. L'ensemble de l'appareillage est stérilisable à la vapeur.

c) L'électrodialyseur. Nous avons choisi le micropilote P1 produit par la S.R.T.I. (Société de Recherches Techniques Industrielles - Buc, France). Il comprend 20 cellules (surface utile $69 \mathrm{~cm}^{2}$ ). Chacune d'elle comporte une membrane conductrice d'anions, greffée d'amines quaternaires, et une membrane conductrice de cations, sulfonée. Les trois circuits hydrauliques sont reliés à des réservoirs de 1,5 litre contenant respectivement le produit à déminéraliser (bac D), le produit concentré en lactate (bac C) et la solution de rinçage des électrodes (bac E, non représenté sur la figure pour des raisons de clarté).

\section{Capteurs - saisie de données}

Les capteurs implantés le long du circuit hydraulique (fig. 1) permettent de suivre en continu les paramètres qui caractérisent le système. Leur fonction est triple : 
24

- donner des informations sur l'évolution des paramètres 24 heures sur montage ;

- appréhender les inter-relations existant entre différents organes du

- permettre de réagir rapidement aux variations d'un ou plusieurs paramètres du système.

a) Capteurs de température (1). Ils permettent la détection d'éventuels points d'« échauffement » dans le circuit hydraulique. Au niveau de l'ultrafiltre, c'est un thermo-couple cuivre/constantan (IONITHERM, France) et dans l'électrodialyseur une sonde de platine couplée à une électrode de $\mathrm{pH}$ (TACUSSEL, France).

b) Capteurs de pression (2). Ce sont des capteurs à membrane affleurante type PE 102 couvrant une gamme 0-10 bars (Techniques Modernes d'Extensométrie - Orgeval, France). Placés en amont et en aval de cartouche d'UF, ils permettent d'évaluer la perte de charge intervenant entre ces deux points, d'où, à débit constant, un suivi du colmatage.

c) Capteurs de débit (3). Nous avons choisi des débitmètres électromagnétiques à induction (Altoflux - Krohne - Romans, France) branchés sur le circuit rétentat (diamètre nominal de $10 \mathrm{~mm}$ ) et sur le circuit perméat (diamètre nominal de $2,5 \mathrm{~mm})$.

d) Capteur de densité optique (4). C'est un prototype mis au point par LALANDE et al. à l'I.N.R.A. de Villeneuve d'Ascq (communication personnelle).

e) Autres capteurs. Des capteurs de $\mathrm{pH}$ (6) sont en place dans le fermenteur et dans le bac D de l'électrodialyseur. Au niveau du fermenteur, il est assorti d'un système de régulation par addition de soude (8). Un capteur de conductivité (5) mis en place dans le bac D de l'électrodialyseur permet de contrôler la déminéralisation du milieu. Des capteurs de niveau (7) assurent la régulation des volumes de produit concentré (bac C) et produit à déminéraliser (bac D). Enfin, intensité et tension aux bornes de l'électrodialyseur sont mesurées respectivement par un ampèremètre (10) et un voltmètre (11).

Toutes les mesures sont converties en signaux électriques 4-20 mA (faciles à véhiculer sur de longues distances). Le stockage, la visualisation et l'exploitation des résultats s'effectuent grâce à une instrumentation programmable par ordinateur. Un standard IEEE 488 (G.P.I.B.) permet la circulation bidirectionnelle entre l'ordinateur et les appareils de mesure. L'essentiel de l'appareillage est constitué par un multimètre et un scrutateur de voies. Un microordinateur (PHILIPS P 2000) permet de réaliser la saisie des données, leur stockage en fichiers ainsi que leur impression et leur représentation graphique.

\section{La fermentation}

a) Milieu de culture. Les bactéries sont cultivées sur perméat de lactosérum doux (PREVAL, France) supplémenté en azote par l'adjonction d'un extrait autolytique de levure industrielle (BIOKAR, France) à la dose de $1 \%$. Le perméat est préparé sur place par ultrafiltration (ROMICON, PM 50, U.S.A.) de poudre de lactosérum reconstituée à $6,5 \%$ dans de l'eau désionisée. 
b) Souches. Nous avons retenu l'association d'un Lactobacillus helveticus et d'un Streptococcus thermophilus (respectivement les souches 303 et 160 de la collection du C.N.R.Z. - Jouy-en-Josas, France), en raison de la synergie existant entre ces deux souches (AcCOLAs et al., 1977) et de leur important potentiel de production d'acide lactique.

c) Conditions de culture. L'entretien des souches s'effectue par repiquages successifs sur milieux semi-synthétiques (MRS, de MAN et al. (1960), pour le lactobacille, M 17, Terzaghi et Sandine (1975) pour le streptocoque). Le dernier repiquage avant inoculation s'effectue sur le milieu de fermentation puis les souches sont inoculées séparément à la dose de $1,5 \%$. La fermentation est conduite à $45^{\circ} \mathrm{C}-\mathrm{pH} 5,5$. Elle a lieu soit en circuit fermé, soit en continu, à volume constant. On définit alors le taux de dilution d (exprimé en heure $^{-1}$ ) comme le rapport du débit d'alimentation en milieu au volume total de fermentation (3 l).

\section{B. Méthodes d'analyses}

\section{Biomasse}

La biomasse totale est évaluée par mesure de l'absorbance à $650 \mathrm{~nm}$ (Spectrophotomètre BECKMAN type Acta MVI). Nous avons établi une relation entre cette mesure et le poids sec de cellules. Une unité de densité optique représente en effet un poids sec de $0,333 \mathrm{~g} \cdot \mathrm{kg}^{-1}$ pour la culture mixte et de $0,400 \mathrm{~g} \cdot \mathrm{kg}^{-1}$ pour une culture pure de Lactobacillus helveticus 303 . Ces valeurs ont par ailleurs été corrélées aux indications du capteur de densité optique. Le taux de croissance $(\mu)$ est calculé comme suit (MONOD, 1942):

$$
\mu=\frac{\log x_{2}-\log x_{1}}{\left(t_{2}-t_{1}\right) \log 2} \text { avec } x_{i}=\text { biomasse au temps } \mathrm{i}
$$

\section{Dosage des sucres}

La méthode employée permet de doser en routine le lactose ainsi que le glucose et le galactose issus de son hydrolyse. Il s'agit d'une chromatographie H.P.L.C. (WATERS ASSOCIATES, Milford, U.S.A.) sur colonne $\mu$-Spherogel carbohydrate (BECKMAN) de $7,5 \times 300 \mathrm{~mm}^{2} .20 \mu \mathrm{l}$ d'échantillon sont élués à $80^{\circ} \mathrm{C}$ par de l'eau (milliQ - WATERS) à un débit de $0,6 \mathrm{ml} \cdot \mathrm{min}^{-1}$. La détection des pics s'effectue par réfractométrie. La durée globale d'analyse est de 25 minutes.

\section{Dosage des acides organiques}

Le lactate de sodium est dosé par H.P.L.C. (même appareillage que précédemment). La séparation s'effectue sur une colonne échangeuse d'ions (AMINEX A6 : silice greffée d'ions $-\mathrm{SO}_{3}{ }^{-}$) de $6 \times 300 \mathrm{~mm}^{2} .15 \mu l$ d'échantillon sont élués par une solution $\mathrm{d}^{\prime} \mathrm{H}_{2} \mathrm{SO}_{4} 10^{-2} \mathrm{~N}$ à température ambiante. $\mathrm{La}$ détection des pics est réalisée par spectrophotométrie d'absorption en ultraviolet $(215 \mathrm{~nm})$ (WATERS, U.S.A.). 


\section{Dosage d'azote}

La teneur en azote total des échantillons est évaluée par microkjeldahl sur une chaîne automatisée TECHNICON (OGG, 1960).

\section{Evaluation de la lyse cellulaire}

La lyse cellulaire, dont une partie est attribuable aux passages répétés des bactéries dans la pompe de circulation du module d'ultrafiltration, est évaluée par dosage de la lactate déshydrogénase (L.D.H.) dans le milieu de culture. En effet, cette enzyme est strictement endocellulaire chez les bactéries lactiques (Garvie, 1980). La quantité relarguée dans le milieu peut donc être directement corrélée au taux de lyse cellulaire. Le dosage est effectué lors de la transformation du pyruvate en lactate dans un tampon triéthanolamine- $\mathrm{HCl}$, $\mathrm{pH} 7,0$, en présence de fructose 1-6 diphosphate. Le suivi est réalisé par mesure de la diminution de l'absorbance à $340 \mathrm{~nm}$ qui résulte de l'oxydation du NADH, cofacteur de l'enzyme, en NAD (ThомAs, 1975).

\section{Etude des caractéristiques rhéologiques du milieu de fermentation}

Nous avons étudié l'effet du cisaillement sur notre milieu de culture par l'établissement d'une courbe $\eta$ (viscosité) en fonction de $\dot{\gamma}$ (gradient de vitesse de cisaillement) à l'aide d'un Rhéomat 30 (CONTRAVES, Suisse) muni d'une cellule à double entrefer spécialement conçue pour les solutions de faible viscosité.

Le type d'écoulement du milieu dans le module d'ultrafiltration est caractérisé par le nombre de Reynolds ( $\mathrm{Re})$ calculé à l'aide de la formule suivante (KESSLER, 1981) :

$$
\mathrm{R}_{\mathrm{e}}=\frac{\mathrm{v} \cdot \mathrm{d} \cdot \rho}{\eta}
$$

$$
\text { avec } \begin{aligned}
\mathrm{v} & =\text { vitesse d'écoulement en } \mathrm{m} \cdot \mathrm{s}^{-1} \\
\mathrm{~d} & =\text { diamètre du tube en } \mathrm{m} \\
\rho & =\text { densité du milieu en } \mathrm{kg} \cdot \mathrm{m}^{-3} \\
\eta & =\text { viscosité absolue en } \mathrm{Pa} \cdot \mathrm{s}
\end{aligned}
$$

\section{Résultats - Discussion}

Les premiers essais ont été conduits en vue d'atteindre trois objectifs :

1. Obtenir une première estimation des performances du système.

2. Mettre en évidence l'effet de quelques paramètres de fermentation sur la productivité des microorganismes. Celle-ci est exprimée en quantité de lactate produite par unité de volume et par unité de temps $\left(\mathrm{g} \cdot \mathrm{l}^{-1} \cdot \mathrm{h}^{-1}\right)$. Ce critère traduit les performances du système. Il fait apparaître la vitesse de la transformation et reflète la concentration en lactate de l'effluent de fermentation. Notre objectif était de produire en un minimum de temps le maximum de lactate et ceci dans un volume d'effluent le plus petit possible. En effet, il faut limiter les quantités à traiter par électrodialyse et les étapes éventuelles de concentration à mettre en œuvre en aval du procédé. Nous aurions également 
pu raisonner en termes de conversion du lactose. Cependant, le faible coût de ce composé et la possibilité de le recycler dans le procédé employé nous ont conduit à privilégier la production de lactate.

3. Tester le fonctionnement et les problèmes techniques éventuels posés par les différents organes du procédé (fermenteur - ultrafiltre - électrodialyseur).

Les résultats présentés ici proviennent de deux essais représentatifs mettant en œuvre le fermenteur et l'ultrafiltre, effectués sur 8 heures (Essai I) et 220 heures (Essai II) et d'un essai sur l'ensemble du dispositif d'une durée de 150 heures (Essai III), ce qui a permis de mettre en évidence la stabilité à long terme de cette fermentation.

\section{A. Performances du système}

La figure 2 présente les résultats concernant la croissance bactérienne et la productivité du système suivant les paramètres de fonctionnement (Essai II).

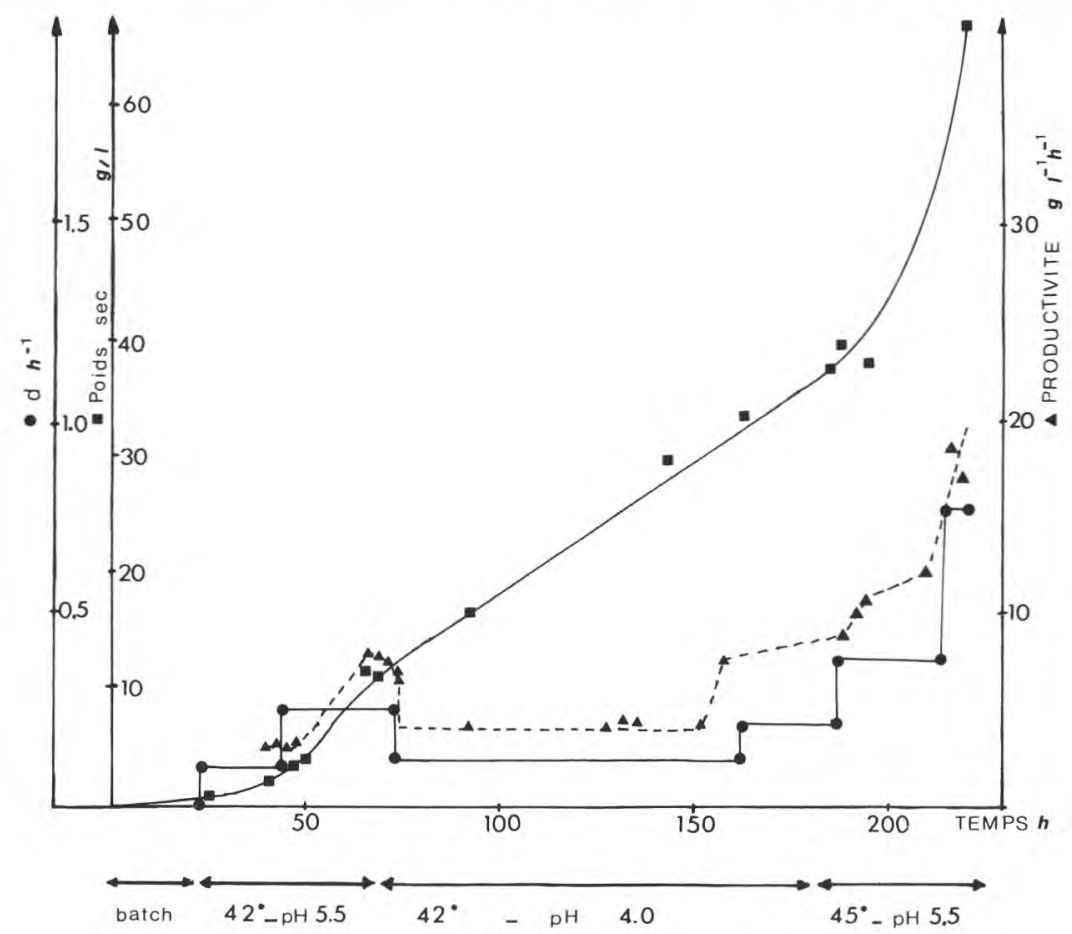

Fig. 2

Essai II : Mélange de Lactobacillus helveticus 303 et Streptococcus thermophilus 160. Suivi de la biomasse, de la productivité en lactate et du taux de dilution (d) au cours du temps.

Run II : Mixed culture of Lactobacillus helveticus 303 and Streptococcus thermophilus 160.

Biomass content, lactate productivity and dilution rate $(d)$. 


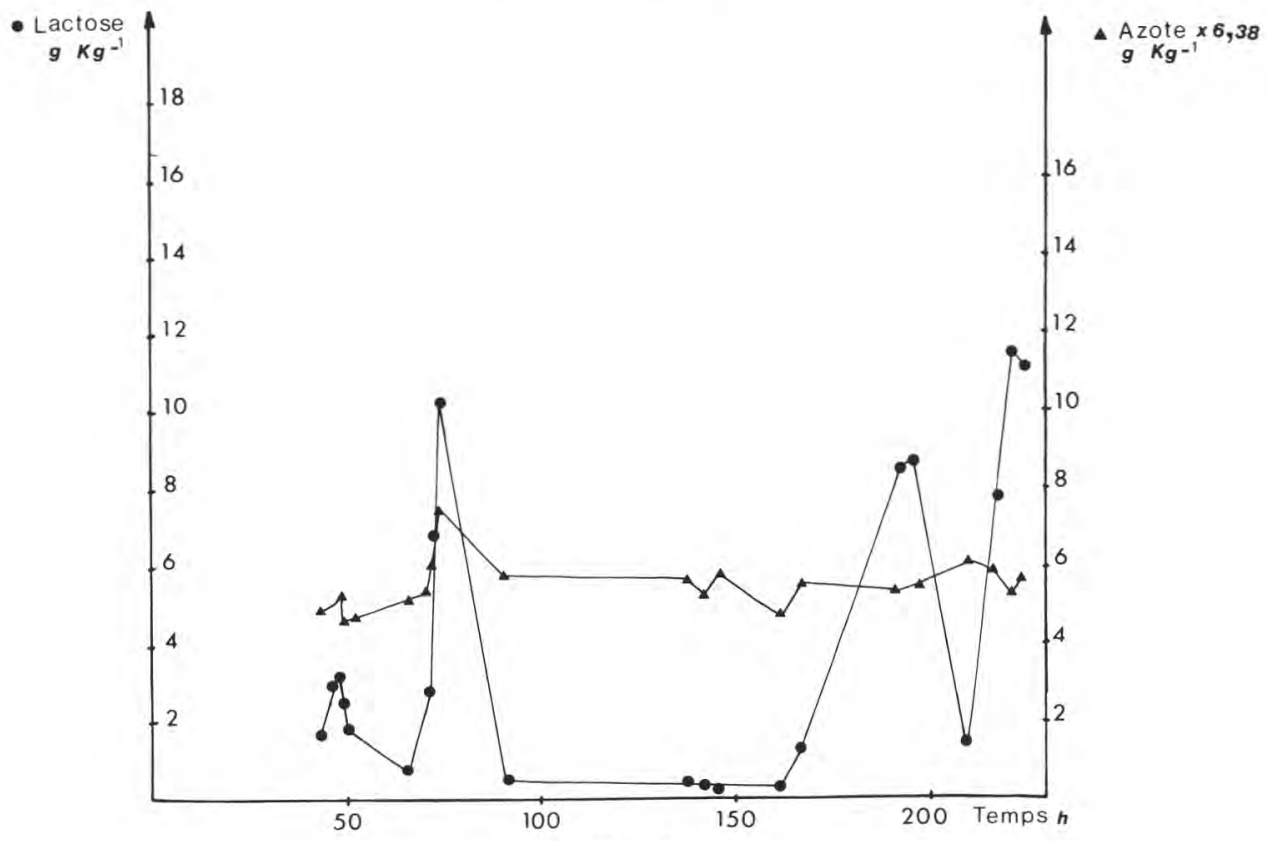

Fig. 3

Essai II : Mélange de Lactobacillus helveticus 303 et Streptococcus thermophilus 160. Evolution des teneurs en nutriments du milieu.

Run II: Mixed culture of Lactobacillus helveticus 303 and Streptococcus thermophilus 160.

Evolution of nutrient content of the broth.

On constate que la culture en «batch » réalisée sur les 24 premières heures se caractérise par une croissance relativement faible (on atteint seulement $1,65 \mathrm{~g} \cdot \mathrm{l}^{-1}$ de cellules en fin de période). Ceci ne peut être relié à un manque de nutriments (fig. 3) mais est probablement dû à une inhibition de la croissance par la présence en trop grande quantité de lactate de sodium dans le milieu (Luedeking et Piret, 1959). Le passage en continu (fig. 2) conduit à une très nette augmentation de la vitesse de multiplication cellulaire (doublement du poids sec en 18 heures) et par l'accroissement de la productivité d'acide lactique. La meilleure efficacité du système se traduit par un rapide appauvrissement du milieu en lactose lorsque le taux de dilution est faible $\left(\mathrm{d}=0,1 \mathrm{~h}^{-1}\right)$ (fig. 2). La levée d'inhibition par le lactate, éliminé en continu dans le perméat d'ultrafiltration peut expliquer ce phénomène.

L'essai II permet également de mettre en évidence l'influence du taux de dilution sur les performances du système ; dans les mêmes conditions de $\mathrm{pH}$ et température (respectivement 5,5 et $42^{\circ} \mathrm{C}$ ), son accroissement de 0,1 à $0,25 \mathrm{~h}^{-1}$ permet de passer d'une productivité de $3 \mathrm{~g} \cdot \mathrm{l}^{-1} \cdot \mathrm{h}^{-1}$ à $8 \mathrm{~g} \cdot \mathrm{l}^{-1} \cdot \mathrm{h}^{-1}$. De même, à $45^{\circ} \mathrm{C}, \mathrm{pH} 5,5$, le passage de $\mathrm{d}$ de $0,38 \mathrm{~h}^{-1}$ à $0,77 \mathrm{~h}^{-1}$ a pour conséquence une augmentation de la productivité de 11 à $18 \mathrm{~g} \cdot \mathrm{l}^{-1} \cdot \mathrm{h}^{-1}$. 
Les effets du $\mathrm{pH}$ et de la température sur les performances du système ont également été mis en évidence à la lumière de cet essai. Nous avons testé pour le $\mathrm{pH}$ les valeurs 4,0 et 5,5, nous avions en effet constaté lors de l'essai I que des valeurs supérieures à 5,5 pouvaient poser d'importants problèmes de contamination lors du fonctionnement en continu du système. Malgré l'intérêt qu'aurait présenté un $\mathrm{pH}$ très acide par rapport à ces problèmes, les mauvaises productivités obtenues à $\mathrm{pH} 4,0$ nous ont incités à conserver la valeur de 5,5. Le passage de la température de 42 à $45^{\circ} \mathrm{C}$ a permis à la fois de limiter les risques de contamination et d'augmenter la productivité. Nous avons donc retenu le couple $\left(\mathrm{pH} 5,5-45^{\circ} \mathrm{C}\right)$ pour la suite de nos travaux.

L'essai III, effectué à $45^{\circ} \mathrm{C}$ et $\mathrm{pH} 5,5$ a permis de faire fonctionner l'ensemble du système sur une période de 220 heures avec une bonne stabilité. Il a montré qu'il était possible d'obtenir un effluent d'électrodialyse purifié très concentré en lactate $\left(130 \mathrm{~g} \cdot \mathrm{1}^{-1}\right)$ (fig. 8 et 9 ).

Le produit obtenu présentait la composition minérale moyenne suivante : $11,8 \mathrm{~g} \cdot \mathrm{l}^{-1}$ de $\mathrm{Na}, 2,05 \mathrm{~g} \cdot \mathrm{l}^{-1}$ de $\mathrm{K}, 0,43 \mathrm{~g} \cdot \mathrm{l}^{-1}$ de $\mathrm{Ca}$ et $0,10 \mathrm{~g} \cdot \mathrm{l}^{-1}$ de $\mathrm{Mg}$. Ce résultat, comparé à la composition du milieu initial confirme la différence de comportement des minéraux lors de l'électrodialyse et, en particulier, la migration plus rapide des monovalents $(\mathrm{Na}, \mathrm{K})$ que des divalents (HigGins et SHORT, 1980).

\section{B. Aspects concernant la fermentation}

\section{Bris cellulaire dans le circuit de confinement}

L'évaluation de la biomasse totale ne reflète pas l'état physiologique des bactéries. Un des points qu'il était important d'étudier était l'influence du passage répété des cellules dans la pompe de circulation de l'ultrafiltre. Le suivi de la lactate deshydrogenase (L.D.H.) relarguée dans le milieu, illustré par la figure 4, semblait la technique la plus aisée pour suivre un éventuel bris cellulaire.

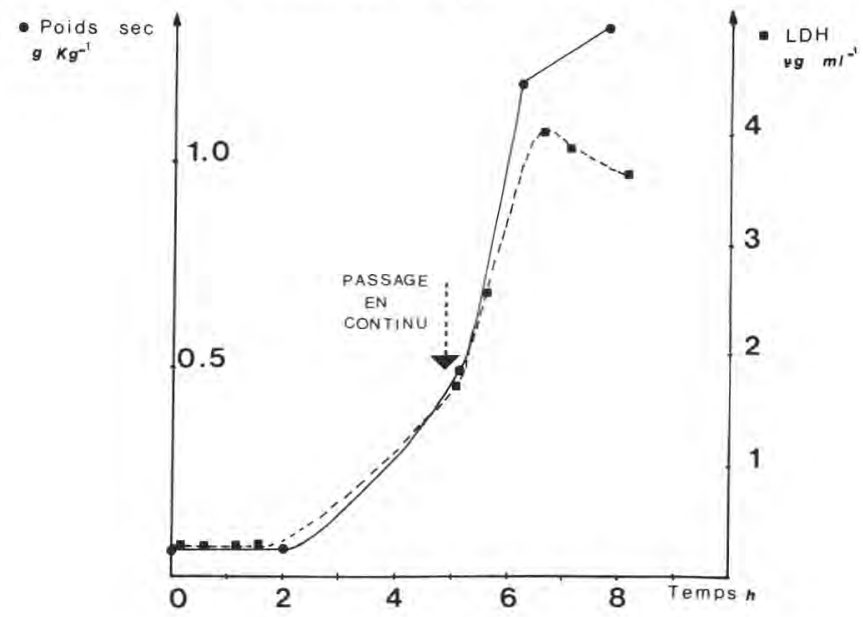

Fig. 4

Evolution de la biomasse totale (-) et de la lactate déshydrogénase exocellulaire (田). Essai 1 .

Total biomass (1) and exocellular lactate dehydrogenase () evolution. Run I. 
L'évolution du poids sec et celle de la L.D.H. dans le milieu au cours du temps sont fortement corrélées. D'autre part, la mise en route de l'ultrafiltre ne provoque pas d'augmentation brutale du relargage de L.D.H. Il semble donc que la pompe n'endommage pas les cellules. Ce résultat obtenu sur un milieu à faible biomasse (de l'ordre de $1 \mathrm{~g} \cdot \mathrm{kg}^{-1}$ ) a été confirmé par la suite lors d'un essai en milieu plus concentré $\left(14 \mathrm{~g} \cdot \mathrm{kg}^{-1}\right)$. Cet essai a également montré que l'augmentation de la vitesse de circulation dans la pompe n'accroissait pas les bris cellulaires.

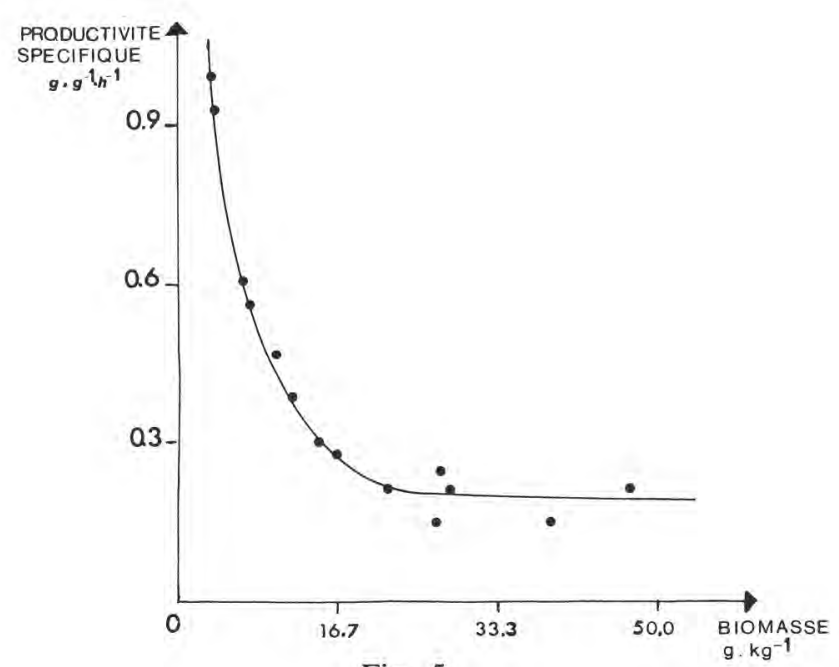

Fig. 5

Influence de la densité cellulaire sur la productivité spécifique (quantité de lactate produite par gramme de biomasse et par heure).

Cell density effect on specific productivity (lactate amount produced per $\mathrm{g}$ of biomass per hour).

\section{Concentration en biomasse et productivité spécifique}

La figure 5 montre que la productivité spécifique (quantité de lactate produite par heure ramenée au g de biomasse présente) est d'autant plus faible que la densité cellulaire dans le milieu est élevée. En particulier, elle décroît très rapidement dans la zone correspondant à des biomasses allant jusqu'à $17 \mathrm{~g} \cdot \mathrm{kg}^{-1}$ puis se stabilise au-delà de cette valeur. Pour une biomasse de $3,3 \mathrm{~g} \cdot \mathrm{kg}^{-1}$, on observe une productivité de 0,9 gramme d'acide lactique par gramme de biomasse et par heure, on passe de 0,6 gramme pour une biomasse de $6,7 \mathrm{~g} \cdot \mathrm{kg}^{-1}$ et enfin au-delà de $16,7 \mathrm{~g} \cdot \mathrm{kg}^{-1}$, on se stabilise à 0,3 , soit 3 fois moins que la valeur initiale. Ce résultat confirme les travaux de Goma et al. (1984) et montre que les phénomènes d'inhibition par le produit ne suffisent pas à expliquer cette chute de productivité. L'hypothèse que nous pouvons formuler à ce stade de nos travaux et en accord avec GomA et al. (1984) est 
qu'il semble exister dans le fermenteur, à haute densité cellulaire, des limitations diffusionnelles qui affectent les transferts de matière et donc modifient la physiologie bactérienne. Il s'avère donc intéressant de se placer dans le fermenteur à une biomasse optimale permettant à la fois une bonne productivité globale et une utilisation maximale du potentiel bactérien. On ne peut pas, de plus, éliminer le risque de l'accumulation dans le milieu, d'un autre composé exerçant un rôle négatif sur la synthèse de l'acide.

\section{Stabilisation de la biomasse - Etats d'équilibre}

Lors des essais conduits avec recyclage total des cellules nous avons constaté qu'il était impossible de stabiliser la biomasse et donc, d'accéder à un état réel d'équilibre. Lors de l'essai III, nous avons fait varier le débit de la purge de biomasse. La figure 6 présente l'évolution du poids sec en fonction du temps. Le début de l'essai a eu lieu à purge fermée. On constate alors une augmentation constante de la biomasse au cours du temps jusqu'à un seuil de $30 \mathrm{~g} \cdot \mathrm{kg}^{-1}$ de milieu; on a ensuite une légère diminution que l'on a pu attribuer à une carence momentanée en lactose puis, après augmentation du taux de dilution, une poursuite de l'évolution jusqu'à $40 \mathrm{~g} \cdot \mathrm{kg}^{-1}$. Il est lors de cette phase, impossible d'atteindre un état d'équilibre. Au bout de 100 heures de fonctionnement, nous avons ouvert la purge de cellules à un débit de $960 \mathrm{ml} \cdot \mathrm{h}^{-1}$; le temps de séjour de la biomasse était alors de 3 heures et $7 \mathrm{mn}$ (soit un taux de dilution de $0,32 \mathrm{~h}^{-1}$ ); nous avons alors constaté une chute rapide de la biomasse due à un «lessivage » du fermenteur (c'est-à-dire une purge cellulaire supérieure à l'augmentation de la biomasse due à la multiplication des cellules). Le taux de croissance des bactéries, $\mu$, mesuré dans les

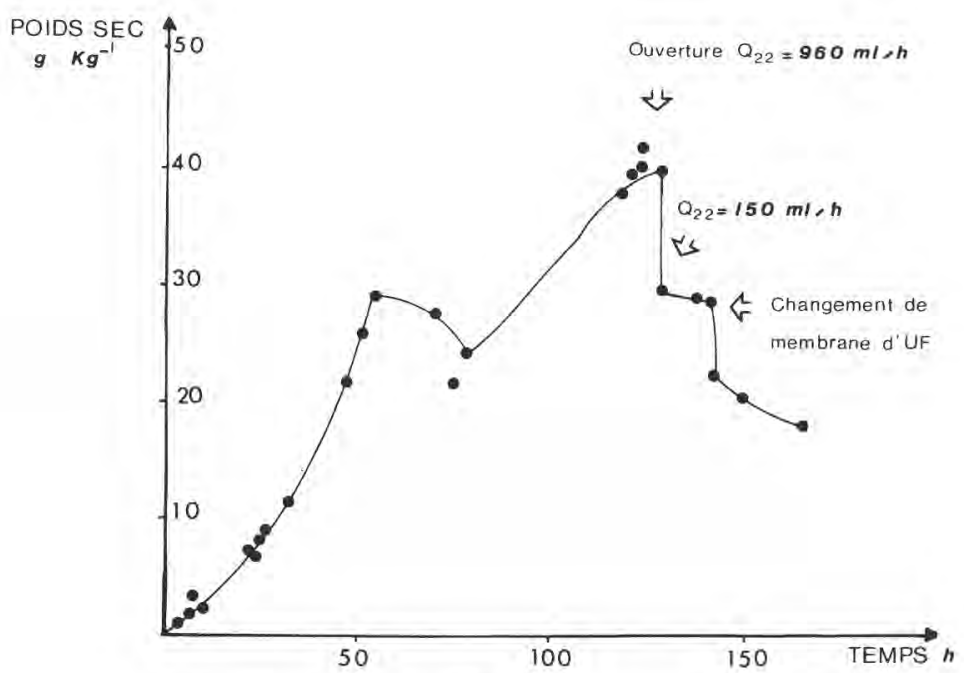

Fig. 6

Essai III : Influence de l'ouverture de la purge cellulaire sur la croissance de la biomasse. Run III : Cells outlet influence on biomass growth. 
heures précédant l'ouverture de la purge cellulaire, était seulement de $0,27 \mathrm{~h}^{-1}$. Ceci révèle un mauvais état physiologique de la culture ; en effet, son taux de croissance maximal sur le milieu considéré a été évalué à $0,98 \mathrm{~h}^{-1}$. On peut expliquer cette mauvaise performance par la déficience du milieu en lactose présent à l'état de traces (moins de $1 \mathrm{~g} \cdot \mathrm{l}^{-1}$ ). Dans un $3^{\mathrm{e}}$ temps, nous avons donc ouvert la purge au débit plus modéré de $150 \mathrm{ml} \cdot \mathrm{h}^{-1}$, soit un temps de séjour de 20 heures pour les cellules. Nous avons alors atteint un état d'équilibre à $37 \mathrm{~g}$ de biomasse par $\mathrm{kg}$ de milieu qui a duré 20 heures et a été interrompu par une modification des paramètres d'ultrafiltration. On peut donc conclure qu'il est impossible de stabiliser la biomasse en travaillant à purge fermée et que seul un taux de recyclage, compatible avec les caractéristiques des bactéries ( $\mu$ en particulier) peut permettre d'atteindre un équilibre.

\section{Aspects concernant l'ultrafiltration}

Nous avons vu que la recirculation continue des cellules dans le circuit d'ultrafiltration n'avait pas d'incidence sur leur viabilité. Au plan technique, le couplage du fermenteur à l'ultrafiltre a cependant posé quelques problèmes.

\section{Problèmes de colmatage de la membrane}

La figure 7 présente l'évolution des débits de perméat et de rétentat au cours du temps. Lors de cet essai, tous les paramètres d'ultrafiltration ont été maintenus constants (vitesse de rotation de la pompe, contre-pression). On observe donc un débit de rétentat constant, fixé ici à $100 \mathrm{l} \cdot \mathrm{h}^{-1}$, ce qui correspond à une vitesse de balayage de la membrane de $0,328 \mathrm{~m} \cdot \mathrm{s}^{-1}$. Parallèlement, on observe durant les 30 premières minutes, une chute rapide du débit de perméat qui se stabilise à $4 \mathrm{l} \cdot \mathrm{h}^{-1}$. Ce phénomène correspond aux modifica-

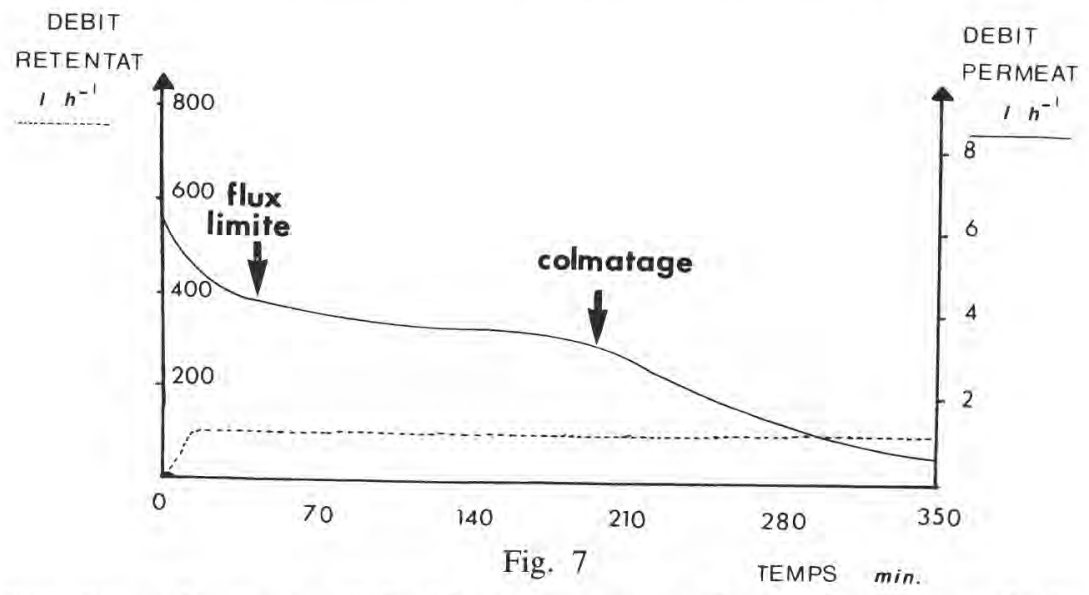

Evolution des performances d'ultrafiltration au cours du temps. Mise en évidence du phénomène de colmatage de la membrane.

Ultrafiltration performances evolution. Demonstration of membrane clogging phenomenon. 
tions des caractéristiques de la membrane (seuil de coupure en particulier) par établissement de la couche de polarisation. Le système est ensuite stable pendant 150 minutes puis on observe une seconde chute de débit plus lente, due au colmatage de la membrane. L'ampleur de ce phénomène peut être reliée au type d'écoulement du fluide dans les membranes. La vitesse de balayage calculée précédemment correspond à un régime laminaire, le nombre de Reynolds $(\mathrm{Re})$ étant très inférieur à 2000 . Lors de l'essai III, nous avons travaillé en régime turbulent ( $\mathrm{Re}$ de l'ordre de 8000 ), ce qui a atténué le phénomène de colmatage (vitesse de balayage de $2,6 \mathrm{~m} \cdot \mathrm{s}^{-1}$ ). Ce résultat est en parfait accord avec tout ce que l'on sait sur l'ultrafiltration. Par contre, il n'existe pas encore de travaux sur l'ultrafiltration de cellules qui puissent permettre des comparaisons de performances de telle ou telle membrane d'ultra ou de microfiltration.

\section{Caractéristiques rhéologiques du milieu de fermentation}

L'étude des phénomènes de transferts de matière dans le fermenteur et l'ultrafiltre implique une caractérisation du comportement rhéologique du milieu. Lors de l'essai I, nous l'avons établi sur un échantillon de milieu contenant environ $30 \mathrm{~g}$ de biomasse par kilogramme, soit une densité cellulaire relativement élevée par rapport aux objectifs.

La viscosité, aux incertitudes de mesures près, est indépendante du gradient de vitesse (entre 0 et $1800 \mathrm{~s}^{-1}$ et égale à $1,8 \mathrm{mPa} \cdot \mathrm{s}$ ), ce qui traduit un comportement newtonien. Ceci permet d'une part d'affirmer que la vitesse de passage dans la pompe n'aura pas d'influence sur la viscosité du milieu et que d'autre part, les bactéries n'excrètent pas dans le milieu de composés de haut poids moléculaire (polysaccharides par exemple).

Ces résultats confirment d'autres travaux (KEMBLowsKi et Kristiansen, 1986) qui ne montrent pas de changement marqué de propriétés rhéologiques des milieux en cours de fermentation, sauf pour les organismes filamenteux ou producteurs de polysaccharides.

\section{Performances d'électrodialyse}

Le couplage de l'électrodialyseur au fermenteur a été réalisé lors de l'essai III, le système complet ayant fonctionné durant 8 heures. La figure 8 montre l'évolution des teneurs en lactate dans les deux compartiments au cours du temps. On observe l'obtention d'une concentration de $130 \mathrm{~g} \cdot \mathrm{I}^{-1}$ après 8 heures de fonctionnement. Les paramètres suivis pendant l'électrodialyse ont été le $\mathrm{pH}$, la conductivité ramenée à $20^{\circ} \mathrm{C}$ et la concentration en lactate dans le bac d'arrivée du perméat (D) ainsi que la concentration en lactate dans le bac produit (C).

Les performances du dispositif sont évaluées par le suivi de la concentration en lactate dans le bac $\mathrm{C}$. On remarque qu'elle augmente constamment au cours du temps mais que sa progression chute très rapidement (de $18 \mathrm{~g} \cdot \mathrm{l}^{-1} \cdot \mathrm{h}^{-1}$ en début d'électrodialyse, on passe à $7,5 \mathrm{~g} \cdot \mathrm{l}^{-1} \cdot \mathrm{h}^{-1}$ après 400 minutes). En principe le flux de passage des ions au travers des membranes est linéairement correlé à l'intensité du courant (I) qui traverse l'électrodialyseur. Nous avons par ailleurs constaté que les paramètres mesurés en $\mathrm{D}$ sont beaucoup plus 


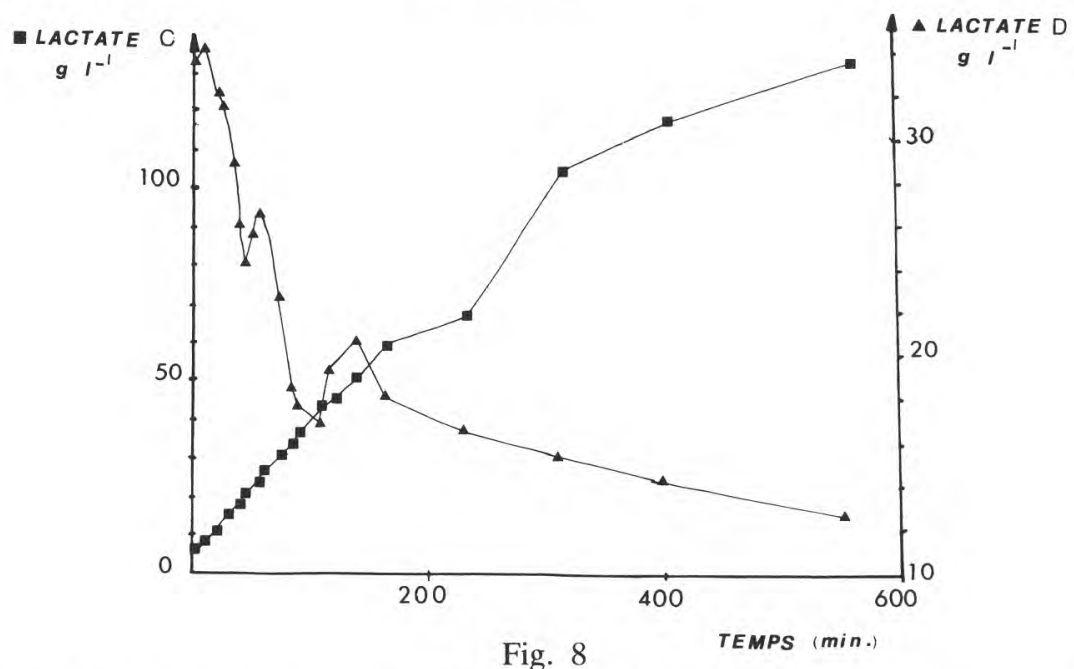

Fig. 8

Essai III : Evolution de la teneur en lactate dans le bac de réception du produit (D) et dans le bac de concentration $(C)$ au cours de l'électrodialyse.

Run III : Lactate content in permeate reception vessel (D) and concentration vessel (C) during electrodialysis.

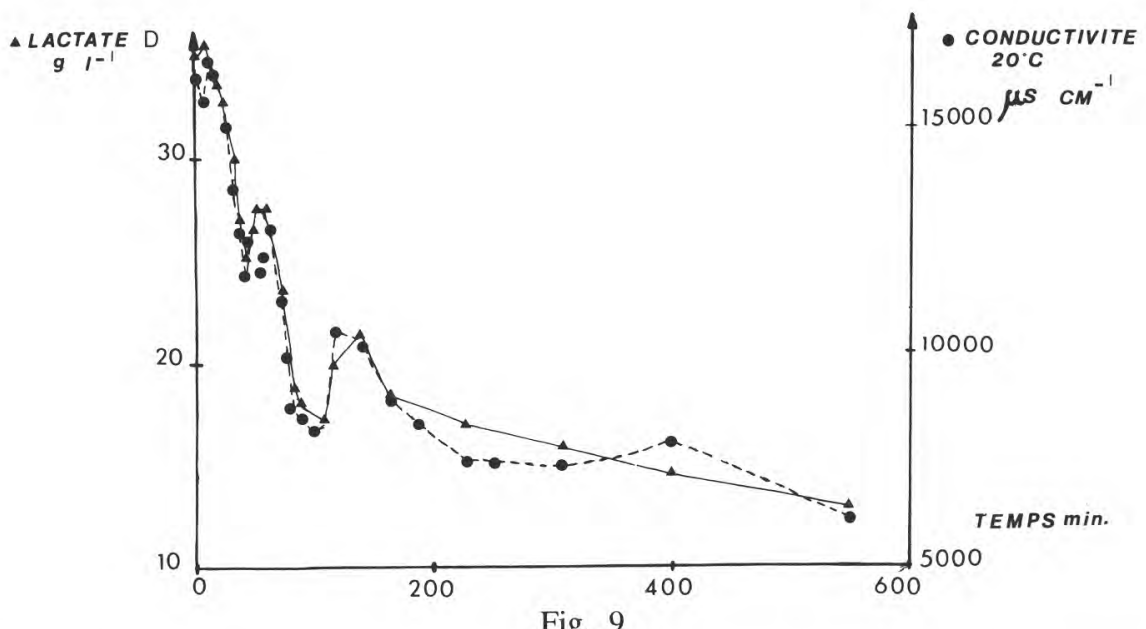

Fig. 9

Essai III : Evolution de la concentration en lactate et de la valeur de la conductivité ramenée à $20{ }^{\circ} \mathrm{C}$ au cours de l'électrodialyse (bac D).

Run III : Lactate concentration and conductivity during electrodialysis (vessel D).

fluctuants que la mesure du lactate dans C. Ces fluctuations sont à relier à l'irrégularité du débit d'alimentation de D. Celle-ci entraîne des variations du volume dans ce compartiment. Les résultats obtenus pour les différentes grandeurs mesurées (fig. 9) montrent une bonne corrélation entre elles. En 
particulier une régression linéaire établie entre la concentration en lactate dans $\mathrm{D}$ et la conductivité corrigée à $20^{\circ} \mathrm{C}(\mathrm{Xi}-20)$ a permis d'obtenir sur 22 points un coefficient de corrélation de 0,988 . On pourra donc par la suite s'affranchir du dosage des lactates dans ce compartiment et suivre l'évolution du sytème en ligne, grâce au capteur de conductivité. Cette étude nous permet donc de tirer quelques conclusions pratiques quant à la mise en cuvre de l'électrodialyseur. Tout d'abord, le travail à intensité constante ne semble pas permettre l'obtention rapide d'états d'équilibre comme nous l'attendions. D'autre part, l'appauvrissement du compartiment $\mathrm{D}$ en ions provoque alors une augmentation de la résistance électrique et donc un accroissement de la différence de potentiels aux bornes de l'électrodialyseur, celle-ci pouvant alors atteindre des valeurs critiques. Enfin, au plan du suivi du procédé, la saisie en continu de la conductivité dans $\mathrm{D}$ et de l'intensité et la tension, aux bornes de l'électrodialyseur devrait nous permettre un contrôle rigoureux du procédé.

\section{Conclusions}

Les résultats obtenus au niveau de la productivité du sytème (environ $20 \mathrm{~g}$ de lactate par litre et par heure à un taux de dilution de $0,77 \mathrm{~h}^{-1}$ ) sont très encourageants pour des essais préliminaires, menés sans souci d'optimisation. Le dispositif présente de nombreux avantages par rapport aux essais menés en réacteurs piston (cellules immobilisées, réacteurs fibres creuses) en particulier en ce qui concerne la régulation des paramètres de fermentation $(\mathrm{pH})$ et le contact substrat-cellule. Les résultats obtenus peuvent être comparés à ceux de Mehaia et Cheryan (1986) qui ont atteint dans le même type de réacteur avec une culture de Lactobacillus bulgaricus des productivités très importantes $\left(80 \mathrm{~g} \cdot \mathrm{l}^{-1} \cdot \mathrm{h}^{-1}\right.$ de lactate). Cependant il faut souligner que la densité cellulaire très élevée à laquelle ils opéraient $\left(60 \mathrm{~g} \cdot \mathrm{kg}^{-1}\right.$ de biomasse posait des problèmes quant aux propriétés physiques du milieu traité ; d'autre part, compte tenu du taux de dilution élevé auquel ils ont travaillé $\left(2 \mathrm{~h}^{-1}\right)$, ils obtenaiént un produit beaucoup moins concentré que le nôtre en lactate $\left(43 \mathrm{~g} \cdot \mathrm{l}^{-1}\right.$ contre $\left.130 \mathrm{~g} \cdot \mathrm{l}^{-1}\right)$. La faisabilité technique du procédé a par ailleurs été démontrée, cependant nous avons dégagé la nécessité d'apporter certains perfectionnements à différents niveaux :

- Sur le plan des risques de contamination, il semble qu'au couple température- $\mathrm{pH}\left(45^{\circ} \mathrm{C}-5,5\right)$ que nous avons sélectionné et dans des conditions d'asepsie correcte, le fonctionnement en continu du dispositif ne pose pas de problèmes. Nous n'avons pas observé de contamination ni au microscope, ni sur isolement.

- En ce qui concerne la mise en œuvre de l'ultrafiltration, nous avons mis en évidence la nécessité de travailler en régime d'écoulement turbulent pour s'affranchir des problèmes de colmatage mais, en tout état de cause, il serait souhaitable de disposer d'un module à double-circuit permettant, en cas de problème d'orienter le milieu à filtrer vers une cartouche propre et de nettoyer automatiquement la cartouche sale. De plus, l'exploration de taux de dilution plus élevés impliquera de disposer d'une surface de membrane plus importante ; en effet, l'ultrafiltration semble être l'étape limitante à l'obtention d'une meilleure productivité du système. 
- Le couplage ultrafiltre-électrodialyseur devra être amélioré de façon à avoir un débit d'alimentation régulier de la cuve D. De plus, l'utilisation sur de longues périodes de l'électrodialyseur nécessiterait la possibilité d'inverser le sens de polarisation des électrodes de façon à éviter le colmatage des membranes, comme cela existe sur les électrodialyseurs industriels.

- En ce qui concerne les caractéristiques à retenir pour le milieu de fermentation, nous avons mis en évidence l'intérêt double de travailler à des densités cellulaires " moyennes» (moins de $17 \mathrm{~g} \cdot \mathrm{kg}^{-1}$ ) : d'une part, cela permet d'avoir une productivité spécifique intéressante et d'autre part les transferts de matière dans le fermenteur se font alors sans limitations diffusionnelles.

Pour la suite de nos travaux, beaucoup de points restent cependant à affiner. En particulier au niveau de l'évaluation économique du procédé plusieurs axes de recherche doivent être développés.

Afin d'optimiser le fonctionnement du système, nos prochains travaux devront permettre d'établir les meilleures conditions pour l'ultrafiltration (augmentation des surfaces de membrane, effet de la viscosité du milieu sur les performances de la filtration). Différents taux de recirculation de la biomasse devront également être testés pour étudier l'obtention des états d'équilibre assurant la meilleure productivité et la meilleure stabilité du dispositif. Nous avons également souligné l'importance de la qualité du produit obtenu, produit dont nous nous proposons de caractériser plus finement la composition. De cette étude découleront ses applications potentielles en l'état ou après d'autres étapes d'aval (purification, concentration) à déterminer.

Enfin, il faut prendre en compte avant toute approche économique du procédé, le coût du milieu traité. Si le perméat de lactosérum est particulièrement bon marché, l'extrait de levure utilisé pour sa supplémentation azotée est prohibitif d'où la nécessité d'une étude concernant la nature et le niveau d'azote à apporter au milieu.

Reçu le 27 janvier 1987.

Accepté pour publication le 15 mai 1987.

\section{Remerciements}

Nous tenons à remercier J.-L. MAUBOIS, Directeur du Laboratoire de Recherches de Technologie Laitière, pour son constant appui ainsi que M. RoIGNANT pour son aide et ses nombreux conseils techniques et informatiques tout au long de ce travail.

Cette étude a été financée par l'association «Bretagne Biotechnologies Alimentaires , par l'Etablissement Public Régional de Bretagne et le Ministère de l'Agriculture. 


\section{Références bibliographiques}

Accolas J.P., Bloquel R., Didienne R., Regnier J., 1977. Propriétés acidifiantes des bactéries thermophiles en relation avec la fabrication de yoghourt. Lait, 57, 1-23.

Boolj C.J. 1985. Use of lactose in the pharmaceutical and chemical industry. J. Soc. Dairy Technol., 38 (4), 105-109.

Burgess K.J., Kelly J., 1979. Alcohol production by yeast in concentrated ultrafiltration permeate from Cheddar Cheese whey. Ir. J. Food Sci. Technol., 3, 1-9.

Champagne C., Boyaval P., 1986. Les cellules immobilisées et l'industrie laitière. Tech. Lait., (1015), 26-32.

CNIEL, 1986. L'économie laitière en chiffres.

Coton S.G., 1980. Whey technology : the utilization of permeates from the ultrafiltration of whey and skim milk. J. Soc. Dairy Technol., 33, 89-94.

DE Boer R., Robbertsen T., 1983. Electrodialysis and ion exchange processes; the case of milk whey. In : Progress in Food Engineering, Cantarelli C. and Peri C. ed., Forster-Verlag AG, Kusnacht. CH, 393-403.

De Man J.C., Rogosa M., Sharpe M.E., 1960. A medium for the cultivation of lactobacilli. $J$. Appl. Bacteriol., 23, 130-135.

Garvie E.I., 1980. Bacterial lactate-dehydrogenases. Microbiol. Rev., 44 (1), 106-139.

Goma G., Durand G., 1986. Nouvelles conceptions de mise en auvre des microorganismes et de bioréacteurs basées sur les cultures à forte densité cellulaire. $11^{\mathrm{e}}$ Colloq. Soc. Fr. Microbiol., Toulouse, 127-143.

Goma G., Uribelarrea J.L., Soucaille P., Minier M., Ferras E., 1984. High cell concentration cultures : physiological factors affecting their achievement and technological solutions. Proc. 3rd Eur. Congr. Biotechnol., Munich, II, 451.

HANSEN R., 1980. Carbery milk products in Ireland produces alcohol from whey. Nordeuropaisk Mejeri Tidsskr., 46, 10-13.

Higgins J.J., ShorT J.L., 1980. Demineralization by electrodialysis of permeates derived from ultrafiltration of wheys and skim milk. N.Z. J. Dairy Sci. Technol., 15, 277-288.

Hongo M., Nomura Y., Iwahara M., 1986. Novel method of lactic acid production by electrodialysis fermentation. Appl. Environ. Microbiol,, 52 (2), 314-319.

Inloes D.S., Taylor D.P., Cohen S.N., Michaels A.S., Robertson C.R., 1983. Ethanol production by Saccharomyces cerevisiae immobilized in hollow-fiber membrane bioreactors. Appl. Environ. Microbiol., 46 (1), 264-278.

Kemblowski Z., Kristiansen B., 1986. Rheometry of fermentation liquids. Biotechnol. Bioeng., $28,1474-1483$.

Ko Y.T., Chipley J.R., 1983. Microbial production of lysine and threonine from whey permeate. Appl. Environ. Microbiol., 45, 610-615.

Kessler H.G., 1981. Food Engineering and Dairy Technology, Verlag A. Kessler, FRG.

LeE H.K., Maddox I.S., 1986. Continuous production of 2.3 butanediol from whey permeate using Klebsiella pneumoniae immobilized in calcium alginate. Enzyme Microb. Technol., 8, 409-411.

Luedeking R., Prret E.L., 1959. A kinetic study of lactic acid fermentation. Batch process at controlled pH. J. Biochem. Microbiol. Technol. Eng., 1 (4), 393-412.

Maubois J.-L., 1982. Les protéines du lactosérum extraites par ultrafiltration. In : Protéines animales, extraits, concentrés et isolats en alimentation humaine. Bourgeois C.M. et Le Roux P. éd., APRIA-Lavoisier, Paris, 172-190.

Mehaia M.A., Cheryan M., 1984a. Ethanol production in a hollow fiber bioreactor using Saccharomyces cerevisiae. Appl. Microbiol. Biotechnol., 20, 100-104.

Mehaia M.A., Cheryan M., 1984b. Hollow fiber bioreactor for ethanol production : application to the conversion of lactose by Kluyveromyces fragilis. Enzyme Microb. Technol., 6, 117-120.

Mehaia M.A., Cheryan M., 1986. Lactic acid from acid whey permeate in a membrane recycle bioreactor. Enzyme Microb. Technol., 8 (5), 289. 
Michelet J., Deschamps A., Lebeault J.M., 1982. L'acide lactique, sa place dans l'industrie. Colloq. Soc. Fr. Microbiol., I.F.P., 39-54.

Monod J., 1942. Recherches sur la croissance des cellules bactériennes. Thèse. Ed. Hermann, Paris.

Morris C.E., 1982. New developments in whey processing. Food Eng., 54, 67-72.

Moulin G., Galzy P., 1976. Une possibilité d'utilisation du lactosérum : la production de levures. Ind. Agric. Alim., 93 (11), 1337-1343.

Moulin G., Malige B., Galzy P., 1983. Balanced flora of an industrial fermentor. Production of yeasts from whey. J. Dairy Sci., 66, 21-28.

OgG C., 1960. J. Assoc. Off. Anal. Chem., 43, 89.

Porter M.C., Michaels A.S., 1970. Applications of membrane ultrafiltration to food processing (Abstract). 3rd Int. Congr. Food. Sci. Technol. "The Science of Survival ».

Prigent Y., Franco A., 1984. Etude d'un fermenteur lactique à membrane. Lait, 64, 217-238.

Ramanathan M., Gaudy Jr A.F., 1971. Steady-state model for activated sludge with constant recycle sludge concentration. Biotechnol. Bioeng., 13, 125-145.

Schwartz R.D., 1986. Biopolymers from whey. Int. Whey Conf., Chicago, U.S.A.

Smith B.R., Mc BeAN R.D., Cox G.C., 1977. Separation of lactic acid from lactose fermentation liquors by reverse osmosis. Aust. J. Dairy Technol., 32, 23-26.

Sonoyama T., 1968. Recovery of products from fermentation processes by ultrafiltration. M.S. Thesis. Department of Nutrition and Food Science. M.I.T., Cambridge.

Stenroos S.L., Linko Y.Y., Linko P., 1982. Production of lactic acid with immobilized Lactobacillus delbrueckii. Biotechnol. Lett., 4, 159-164.

Terzaghi B.E., Sandine W.E., 1975. Improved medium for Lactic Streptococci and their bacteriophages. Appl. Microbiol., 29 (6), 807-813.

Tномаs T.D., 1975. Tagatose 1-6. Diphosphate activation of lactate-dehydrogenase from Streptococcus cremoris. Biochem. Biophys. Res. Commun., 63 (4), 1035-1042.

Topiwala H.M., Khosrovi B., 1978. Water recycle in biomass production process. Biotechnol. Bioeng., 20, 73-85.

Tuli A., Sethi R.P., Khanna P.K., Marwaha S.S., Kennedy J.F., 1985. Lactic acid production from whey permeate by immobilized Lactobacillus casei. Enzyme Microb. Technol., 17 (4), 164-168.

WANg D.I.C., SinSKey A.J., ButTerworth T.A., 1970. Enzyme processing using ultrafiltration membranes. In: Membrane Science and Technology, J.-E. Flinn, ed., Plenum Press, N.Y, 98-119. 